\title{
Complementary methods for solving crystal structures of intergrown nano-phases
}

\author{
$\underline{\text { Huifang Xu}}{ }^{1}$, Shiyun Jin ${ }^{1}$, and Seungyeol Lee ${ }^{1}$ (hfxu@geology.wisc.edu) \\ ${ }^{1 .}$ Department of Geoscience, University of Wisconsin-Madison, 1215 W. Dayton St., Madison, \\ Wisconsin 53706, United States
}

Understanding crystal structures of nano-phases and nano-precipitates are important to elucidate formation mechanism and subsolidus reactions of minerals and their host rocks. Single-crystal or powder XRD method has limitation in solving these problems. Obtained structure may be an artifact resulted from single phase assumption for the intergrown nano-phases. For instance, a "monoclinic laihunite superstructure (laihunite-3M)" is an artifact from intergrowth of laihunite (1M) and an orthorhombic 3-layer phase. Aberration-corrected Z-contrast imaging can provide chemical images with sub-Å resolution. Scanning transmission electron microscopy (STEM) uses a high-angle annular darkfield (HAADF) detector to give the most highly localized 1s Bloch state imaging. Z-contrast images are HAADF images with atomic resolution. Multiple diffraction effects that appear in high-resolution transmission electron microscopic (HRTEM) imaging can be eliminated or minimized in Z-contrast imaging, because Z-contrast imaging uses non-coherent and elastically scattered electrons at high scattering angle. Using a HAADF detector and annular bright-field (ABF) detector, both a Z-contrast image and an annular bright-field image can be obtained simultaneously. The intensity of Z-contrast images is dependent on the atomic number of atom and occupancy in the site through $Z^{n}$. The power $n$ $(\sim 2)$ depends on experimental conditions. Local composition and occupancy may be obtained from measured intensities. We can obtain positions of atoms directly over a large range of thickness to identify columns of different atoms and their occupancies along the beam direction. Crystal structures of intergrown nano-minerals and nano-precipitates within host phases can be solved by combining the Zcontrast imaging, ab-initio calculation using density functional theory (DFT), and / or single-crystal XRD methods. The complementary methods allow us to solve crystal structures of nano-phases with ordered vacancies in Fe-bearing olivine host, a new monoclinic $\mathrm{Fe}_{3} \mathrm{~S}_{4}$ nano-phase in Fe-sulfide, and nano-precipitates (or G. P. zones) within common rock-forming minerals of orthopyroxenes and alkali feldspars. 